\title{
Towards a Typology of Relevance
}

\author{
Kaveh Mohajeri \\ Lund University \\ kaveh.mohajeri@ics.lu.se
}

\author{
Dorothy E. Leidner \\ Baylor University and Lund University \\ dorothy_leidner@baylor.edu
}

\begin{abstract}
This essay presents a speculative work on making distinctions among different equally valid types of research relevance. The work is innovative not only because it departs from the extant monistic perspectives, where only narrow forms of relevance are acknowledged, towards a pluralist perspective, but also because it recognizes and accounts for the plurality in the perceptions of relevance among different stakeholder groups of the same research. The pluralist perspective draws on the notion of "empowerment," widely employed in such domains as education and social work, and suggests that relevant research in fact can be understood as empowering research to which different stakeholder groups can relate in one way or another. Two analytical dimensions are identified in relation to the notion of "empowerment," and are used in order to demonstrate four general types of relevance that can be achieved in IS research.
\end{abstract}

\section{Introduction}

Scholarly discourse concerning research value are dominated by debates on methodological rigor and theoretical contribution. Those who have had papers rejected from major journals often read reviewer comments suggesting that their work either lacks appropriate methodological rigor or fails to make significant theoretical contribution, or both. Particularly in the case of theoretical contribution, reviewers and editors are often content to simply state that the work does not make adequate theoretical contribution without proffering what such contribution might entail. Our conferences often entertain with panels of highly regarded senior scholars opining differing perspectives on the importance of various method and theory requirements expected by our major journals, and editorials and commentaries continue to project differences in opinion.

Similar debates have occurred with regards to the issue of research relevance, with relevance often seen as incompatible with either methodological or theoretical rigor. Among the more widely cited perspectives on rigor versus relevance have been the 1999 MIS Quarterly essays on the topic featuring such noted scholars as Bob Zmud, Lynda Applegate, Tom Davenport, Lynne Markus, Allen Lee, Izak Benbasat, John King and Kalle Lyytinen. The scholars offered divergent views as to the causes of the lack of relevance within the IS field, the best means of pursuing research of value to practice, the best role models to emulate in the design of practicerelevant research, and the target audience for relevant research. Interestingly, neither in this seminal debate nor in subsequent writings on the topic, has the notion of "relevance" been subjected to nearly the same scrutiny as have the notions of methodological rigor or theoretical contribution. We attribute this in part to the fact that relevance has often been positioned as an antithesis to rigorous theory and method. If a choice has to be made, then the legitimacy of the field demands that theory and method be favored over relevance. However, we align with those who challenge the validity of such dichotomy and wish to advance a field that can, and should, be at once highly rigorous but also highly relevant. Toward this end, one must dig much deeper into the notion of relevance, to unleash the potential of this notion to better shape our view on research value and to enable scholars to pursue multiple forms of relevance rather than a narrow, singularly defined concept of relevance obtainable by a few. In this light, the purpose of this essay is to address research relevance not merely from the perspective of what it is, but also from the perspective of the plurality of forms that it may take. More specifically, this essay will propose a typology of research relevance (henceforth, relevance). 


\section{On relevance}

In the IS field, much of the thoughts, papers and debates on relevance have been concentrated on notions such as providing a single sentence definition of relevance, arguing for or against the lack of relevance, and recommending ways by which relevance could be improved, sometimes even from the standpoint of only one research philosophy (e.g., positivism in the case of Benbasat and Zmud [3]). However, relevance in its own right has not been a serious topic of research and little in-depth IS research has been done on the nature and complexity of this concept. In the aforementioned MISQ 1999 debate on "rigor vs. relevance," relevance is often treated in an ontologically monistic manner, as if "true" relevance can have only one form. This situation also holds true for most other major works concerned with relevance [e.g., 7, 14, 24, 26, 28 and 29]. Consequently, a typology of relevance, which would consider different equally important types or forms of relevance, has been largely absent or, as damagingly, highly implicit in the literature.

A typology of relevance, in particular, and a much deeper understanding of the relevance phenomenon, in general, are long overdue in IS and other business disciplines [9, 16, 21, 22, 28, 29]. Developing a typology of relevance is a very significant task not only because of its role in conveying the relevance phenomenon, but also because it is an important step towards a more complete awareness of the diversity of IS research stakeholders, something which has been a matter of concern for at least the past two decades. For instance, Davenport and Markus [4] argue that IS research should not only target practitioners and managers, but also students. And in more recent debates, society is considered another important stakeholder of IS research [7, 13].

In order to propose a typology of relevance, one must first adopt a pluralist ontological perspective wherein the distinction of different forms of relevance is plausible. For that purpose, we draw on the general notion of "empowerment" as adopted in such domains as education and social work and develop an empowerment perspective of relevance. In the next section of this essay, we first review three existing perspectives, where views given with respect to the issue of relevance are narrow or monistic in one way or another. Then, we lay the groundwork for our typology of relevance by elaborating on our proposed perspective, i.e., the empowerment perspective. In the fourth section, we offer our typology of relevance. Subsequently, in the fifth section, we discuss three major implications arising from the empowerment perspective as well as from our identification of different types of relevance. Finally, we conclude with a summary of major arguments made throughout the essay and provide suggestions for future research.

\section{Different perspectives on relevance}

Albeit difficult to draw firm lines between different perspectives on, or approaches towards, perceiving relevance, our review of the extant accounts revealed at least three distinct, yet partially overlapping, ontological perspectives. These perspectives are ontological in the sense that they hold varying assumptions about the reality and nature of relevance.

\subsection{The applicability perspective}

The most common perspective on relevance is what we refer to as the applicability perspective. According to this perspective, relevance is primarily concerned with the immediate usefulness or utilization of research in practice by practitioners. This perspective is prevalent in early writings on relevance.

A seminal instance of this perspective in IS is manifested in the work by Benbasat and Zmud [3]. Drawing on Astley and Zammuto [1], Benbasat and Zmud [3, p. 9] presume that central to relevance is the idea of "direct utilization." In other words, they imply that "true" relevance emerges only when what they call the outputs (i.e., tools, techniques and practices) of academic work hold "immediate and real value to practice” [3, p. 9]. Subsequent scholars extended the perspective given by Benbasat and Zmud [3]. For instance, Rosemann and Vessey [26] build on Benbasat and Zmud [3] in proposing a research relevance improvement approach called “applicability check.” Their approach encourages IS researchers to conduct focus group or nominal group sessions wherein practitioners' views could be solicited on the importance, accessibility and suitability of such research artifacts as theories, models, processes and so forth.

The applicability perspective maintains that "true" relevance essentially has only one form, that of satisfying the needs of a singular stakeholder group of IS research, namely, the practitioners. Moreover, as Davenport and Markus [4] point out, the applicability perspective, as portrayed in Benbasat and Zmud [3], often advocates for only one form of academically accepted relevant research, i.e., "applied theory" research, where the conception of relevance only goes so far as to suggest that research 
should apply academic theories to practical problems in order to produce seemingly applicable results or prescriptions. Such a conception of relevance is limited for several reasons, not the least of which is it stops short of including the additional form of relevance that might emerge when research-based prescribed courses of action, tools, and interventions are retrospectively evaluated in terms of their actual usefulness or consequences in the real world. Thus, although helpful in presenting and legitimizing academic work that seeks to influence practice, the applicability perspective offers an ontologically monistic view where the primary valid form of relevant research is applicable research and the relationships between IS researchers and practitioners is merely that of producer-consumer.

\subsection{The knowledge production-transfer perspective}

Like the applicability perspective, the knowledge production-transfer perspective of relevance advocates the notion of research producersconsumers. However, unlike the applicability perspective, the knowledge production-transfer perspective recognizes that diverse groups can be the audience of IS research. According to the knowledge production-transfer perspective, the role of IS researchers is to produce new knowledge and to transfer this new knowledge to academic and nonacademic customers/clients through appropriate outlets/channels. Relevant knowledge is knowledge that is readily transferred.

Because of the emphasis on new knowledge, the choice of a research topic plays a critical role in achieving relevance for scholars taking the knowledge production-transfer perspective of relevance. The means of scientific knowledge transfer is the other key factor in achieving relevance. Nevertheless, the literature promoting the knowledge production-transfer perspective typically presents the scientific knowledge transfer factor as more critical than the choice of research topic in achieving "true" relevance. For instance, Dennis [5, p. 40] calls for the development of "new vehicles to promote, nurture, and validate" the dissemination of IS knowledge, while maintaining that IS researchers "have done a good job" in terms of the creation of new knowledge. Straub and Ang [29] also emphasize the need for more empirical research on "knowledge transference," concluding that "the scientific evidence to date strongly supports the contention that IS scholars are frequently and consistently studying key practitioner issues” [29, p. v] but perhaps not effectively transferring these to practice. In another work, Gill and Bhattacherjee [10], drawing on the lens of the informing sciences, aim to address what they call the "informing challenge" facing the IS field, arguing that prior commentaries have sufficiently examined the challenge pertaining to what IS researchers should be researching [10, p. 217].

The point we make here is that the works and commentaries maintaining the knowledge production-transfer perspective, wittingly or unwittingly, tend to frame relevance almost solely as a matter of scientific knowledge transfer centered around such important issues as research accessibility, visibility and readability. The way Gallivan and Aryal [8] define relevance, in fact, can be deemed an extreme case of such framing. Gallivan and Aryal [8, p. 1] define relevance as "the mention of IS research and IS researchers in mainstream business magazines and newspapers."

In our view, the knowledge production-transfer perspective, while recognizing a wider range of IS research stakeholders (i.e., academics, practitioners and students) compared to the applicability perspective, deviates towards what we call the consumerization of IS research. We believe that framing the relationship between IS research and its stakeholders as being mere "producer-consumer" hinders the IS field from functioning at a much higher level where the goal, as Lee [16] suggests, is not merely to cater to the whims of research "consumers/clients," but also to criticize, educate and change them.

The knowledge production-transfer perspective, though important in encouraging researchers to ponder ways to transfer their scholarly insights to the outer world, nevertheless offers a monistic and limited view of relevance not only in that it reduces relevance to the issues of research accessibility and readability, but also because, in its conception of relevance, it does not account for the retrospective evaluation of actual real world consequences of IS research after it has been "read" or "accessed." In other words, the knowledge production-transfer perspective seems to view relevance as emerging when a research topic is interesting for the audience and, more importantly, when the audience is able to access and read the research, regardless of whether the audience found value or insight in the research.

\subsection{The value perspective}

Associated with a fairly recent discourse, the value perspective seeks to understand not just the potential for research to be applicable in, and 
transferable to, practice, but also the actual impact such research makes. Even though the value perspective addresses a phenomenon broader than mere relevance, it has ramifications as to how one may approach relevance.

Central to the value perspective is the notion that IS research can be of value to various stakeholders. However, the terms "value" and "stakeholders," have not been necessarily understood in the same way by different authors and panelists. In their panel discussion, Benamati et al. [2] seem to approach the issue more from the angle of shrinking IS major enrollments and departmental budgets, and, therefore, argue for the necessity of the IS field to again "demonstrate its value within the business college" [2, p. 658]. Desouza et al. [7], on the other hand, focus more on the value of IS research for society. They posit that the classic "rigor vs. relevance" discourse is not capable of accommodating the challenges involved in addressing pressing societal problems. Desouza et al. [7] believe that in order for IS research to have a significant impact on society, it must not only be rigorous and relevant but it must also address the two particular concerns of reverberation and responsibility. Perhaps the most seminal work manifesting the value perspective is Hassan [12]. To Hassan [12], the notion of the value of IS research is in fact linked back to the relevance issue through a historical course of developing ideas and debates. Hassan [12] points out that "the relevance issue was followed by the concern for the field's identity and legitimacy, which evolved into the question of the field's core" [12, p. 802]. This entire historical development of ideas and concerns is understood by Hassan [12] as a manifestation "of an underlying problem in the degree of importance or worth of the product of IS research" [12, p. 802]. Hassan [12] suggests that "the value that IS research provides comes from addressing the questions that other disciplines have not addressed or are incapable of addressing” [12, p. 811]. In other words, Hassan [12] views the notion of the value of IS research primarily as a matter of the distinctiveness of IS research topics and products, and suggests that "only when the field has its own identity and theoretical foundations can it be expected to influence ... industry practice in a significant way" [12, p. 802].

We recognize the importance of the general idea underlying the value perspective, namely, that significant research can often extend beyond what is traditionally known as relevant research. One major idea that we share with those who advocate the value perspective is that the broader and richer notion of "research stakeholders" should be considered in lieu of some other narrower notions such as "research audience," "research customers" or "research consumers," when thinking about the relevance of IS research.

Nevertheless, in spite of its proclaimed emphasis on the need for IS research to serve all research stakeholders, the value perspective seems to be primarily driven by a self-serving desire to create or maintain values that would save the IS field from an anticipated extinction, rather than, as Grover [11] suggests, a willingness to also create value "for a broader constituency of external stakeholders” [11, p. 840]. Perhaps ironically, the works presenting the value perspective largely fail to account for the fact that different stakeholder groups might relate to IS research differently and thus have different perceptions as to what valuable IS research is. Original, active and exposing research, as Hassan [12] indicates, might capture what IS researchers recognize as valuable research, but do those qualities necessarily reflect what, for instance, IS practitioners or IS students perceive as valuable, hence relevant, IS research?

Recognizing the ontologically narrow conceptions of IS research relevance presented in the past, the perspective we propose next can be understood as a general case of which the value perspective, in particular, is a specific instance. Under our proposed perspective, we consider the value of IS research arising not only from the intrinsic qualities that IS research should bear, but also, and more importantly, from the quality of the manner by which diverse stakeholder groups relate to IS research. In other words, rather than focusing on the questions and/or products of IS research, we argue for a focus on the people who appertain to IS research in one way or another.

\subsection{The empowerment perspective}

The empowerment perspective entails our proposed ontological view in regards to relevance. We posit that relevant research can in fact be understood and conceptualized as empowering research.

We draw on a particular conception of empowerment that has long been theorized and used in the education and social work domains, among others [18, 19, 27]. Within those domains, the notion of empowerment refers to a particular form of practice in such areas as educational program evaluation or social service that engages, and gives power and decision-making authority to, all stakeholders with the aim of improving their life experience and well-being in some manner. In more precise terms, Lincoln and Guba [19], for instance, 
argue that an empowering educational program evaluation is an evaluation that "involves all stakeholders from the start, that honors their inputs, that provides them with decision-making power in guiding the evaluation, [and] that attempts to empower the powerless and give voice to the speechless” [19, p. 24].

The notion of empowerment, however, has not remained limited to non-academic practice. It has found its way into the academic research practice as well. In particular, the notion of empowerment has been widely utilized to formulate different views with respect to rigor in non-positivist qualitative research. For instance, Manning [20] explores the "ways to approach purposeful, contextual, and plausible [research] studies” [20, p. 93] using ideas related to the notion of empowerment. Or, Rodwell [25], in developing the rigor aspects of an inquiry mode she calls "constructivist research," employs the notion of empowerment and methodological ideas related to it offered by Lincoln and Guba [19]. Other examples include Kvale [15] and Opie [23].

As for our aim in the current essay, we opine that the notion of empowerment is instrumental in distinguishing among different types of relevance. Under the empowerment perspective, we argue that relevance is primarily a matter of how diverse stakeholder groups relate to IS research, and hence arguably, of how IS research improves the life experience and well-being of those groups in varying ways. Research in IS might be conducted for different purposes and for the benefit of different stakeholder groups, ranging from fulfilling researchers' own inner selves and personal quests to creating instrumentally useful artifacts, and from interrupting patterns of power to building theories that stimulate people to think, act or change. In all such scenarios, however, one common attribute can characterize the manner in which IS research affects people's lives: empowerment.

Empowerment, as implied above, is not a monolithic concept. In other words, an empowering research (or non-academic practice) can affect the lives of its own different stakeholder groups in different ways. A pertinent question then becomes: what are some different ways in which an empowering research (or non-academic practice) can affect its own stakeholders' lives? To answer this question, one does well to consult the work by Lincoln and Guba [19], wherein the authors introduce the "unique criteria of authenticity." The rationale behind introducing the authenticity criteria, as Lincoln and Guba [19] indicate, is to provide a device capable of assessing how well a certain educational program evaluation empowers all the involved stakeholders under the real world conditions of "multiple value structures, social pluralism, conflict rather than consensus, accountability demands, and the like” [19, p. 19]. Because empowering all involved stakeholders will not happen in like fashion, Lincoln and Guba [19] propose that the type(s) of empowerment effect(s) originating from a program evaluation effort may be identified using the authenticity criteria. In this essay, we are particularly interested in the following four authenticity criteria suggested by Lincoln and Guba [19, pp. 22-24]:

- Ontological Authenticity: When a program evaluation effort empowers its stakeholders by raising their consciousness in regard to, for instance, contextual forces "that has had the effect of political, cultural, or social impoverishment," or with respect to "some set of complexities previously not appreciated at all, or appreciated only poorly."

- Educative Authenticity: When a program evaluation effort, in addition to being conducive to a better level of consciousness in regard to a phenomenon, also empowers the stakeholders to come to an appreciation of each other's different types of consciousness, which are rooted in different perspectives and value systems.

- Catalytic Authenticity: When a program evaluation effort, in addition to the preceding two types of empowerment effect, can affect its own stakeholders' lives by facilitating or stimulating action.

- Tactical Authenticity: When a program evaluation effort is not only empowering because it stimulates action, but also empowering because the action taken results in desired changes in the eyes of stakeholders.

The plurality of the ways in which the empowerment effect can transpire, as shown above, has major ramifications for our conceptualization of relevance. One ramification is that just as the empowerment effect can take varying forms, research studies can also be relevant (i.e., have empowerment effect) in different ways. Therefore, holding to the empowerment perspective, one can reasonably argue that all the limited forms of IS research relevance (e.g., to aid practitioners in their problem solving, to address pressing societal problems, etc.) taken as primary forms under the other three competing perspectives (i.e., the applicability, knowledge production-transfer and value perspectives) are indeed specific instances of the general case of empowerment. 


\section{A typology of relevance}

We believe at least two distinct dimensions of the notion of empowerment are discernable when considering the four authenticity criteria originated by Lincoln and Guba [19]. We call these two dimensions empowerment subject matter and empowerment focus.

In our view, the empowerment subject matter dimension involves two particular instantiations: consciousness and action. Consistent with the usage of the terms by Lincoln and Guba [19], consciousness refers to one's subjective awareness or understanding, whereas action refers to one's doing in real world. In the cases of ontological authenticity and educative authenticity, the stakeholders' consciousness is subject to empowerment. The empowerment is either in the shape of raising stakeholders' consciousness in regard to a phenomenon (i.e., as described in the ontological authenticity criterion), or raising their consciousness in regard to each other's different types of consciousness (i.e., as described in the educative authenticity criterion). However, in the cases of catalytic authenticity and tactical authenticity, the subject matter of empowerment is different. It is action.

The way in which the empowerment subject matter, whether consciousness or action, is dealt with depends on another dimension, namely, the empowerment focus. Two specific instantiations of this dimension can be identified when speculating on the authenticity criteria: stimulation and adequacy. Stimulation refers to the mode where empowerment effect transpires when people get motivated to think or act, whereas adequacy refers to the mode where empowerment effect transpires through ensuring that the motivated thought or action develops towards a satisfactory state or acceptable quality. We propose that the empowerment focus is stimulation in the cases of ontological authenticity and catalytic authenticity. In other words, stimulation here deals with motivating either a higher level of consciousness (i.e., as described in the ontological authenticity criterion), or a new course of action (i.e., as described in the catalytic authenticity criterion). However, when the empowerment focus is adequacy, the goal is either to make sure that the stakeholders' consciousness is adequately enriched through their understanding each other's different, and perhaps competing, modes of consciousness (i.e., as described in the educative authenticity criterion), or to ensure that new courses of action are not merely stimulated among stakeholders, but that those courses of action are also effective (i.e., as described in the tactical authenticity criterion).

Drawing on the two dimensions of the notion of empowerment discussed above, we derive a typology of relevance (as shown in Figure 1). Although the number of relevance types matches that of the authenticity criteria, and our language in explaining those relevance types is inspired by Lincoln and Guba [19] in their formulation of the authenticity criteria, we do not intend to make a parallel between the two concepts of authenticity and relevance. In other words, we only engage with the typology of empowerment (and hence, relevance) implied in the work by Lincoln and Guba [19], and not with the authenticity criteria.



Figure 1. A typology of relevance

According to Figure 1, we propose that relevance can be understood as having four general types. These relevance types are general in the sense that some more specific variants may exist under each type:

- Cognitional Relevance: Research bearing this type of relevance is concerned with the empowerment subject matter of consciousness and the empowerment focus of stimulation. Such research is thus characterized by its tendency to generate new ways of understanding the nature and reality of existing phenomena or to raise awareness with regard to the nature and reality of new phenomena. The form of relevance advocated under the knowledge productiontransfer perspective might thus appear to be a variant of this general type of relevance.

- Edificational Relevance: Research is considered to bear this type of relevance when its empowerment effect is in the form of providing educative value. Research is of educative value 
when it offers an opportunity for apprehending, discerning and understanding competing perspectives and value systems in regard to a phenomenon under investigation. The form of relevance presumed by Lee [16] in his suggested model of "critical social theory," or the form that he and also Davenport and Markus [4] argue to be invoked through IS academics' role as teachers, might be considered as variants of this type of relevance.

- Inspirational Relevance: Research is considered to bear inspirational relevance when it is understood as feasibly facilitating or stimulating real world action. This type of relevance may, for instance, emerge in research that offers prescriptions for taking a course of action or designing an artifact. The form of relevance conceptualized under the applicability perspective may therefore refer to a variant of this relevance type.

- Effectuational Relevance: Research is considered to bear this type of relevance if there is evidence that its prescribed courses of action or artifact designs have actually been beneficial or effective in one way or another. Some modes of inquiry such as action research or design research present potential for producing effectuationally relevant outcomes.

\section{Discussion}

The identification of four types of relevance in the preceding section serves as a speculative effort aiming to contribute to a deeper grasp of the relevance phenomenon. The significance of this speculative effort however can be better acknowledged if one also considers the following three major implications.

\subsection{Plurality in the perceptions of relevance}

To draw on the notion of empowerment not only paves the way to offer a pluralist view on relevance, but also enables one to account for how different stakeholder groups, including the researcher(s) conducting the research, might perceive the same research as being empowering, hence relevant, in different ways. The impression we have received from the extant perspectives found in the literature is that research is presumed to be relevant to all its diverse stakeholder groups in the same manner. Looking through the empowerment perspective, however, provides the necessary analytical capacity to grasp and explain the plurality in the perceptions of relevance among different stakeholder groups of the same research study. We believe the empowerment perspective gives a view on relevance from the other side, i.e., from the side of people who (can) have something at stake in the conduct of a research project, and would or should feel being empowered in different equally valid and important ways by that research. Considering our proposed typology of relevance, this thus means that while a given research might bear, for instance, cognitional relevance in the eyes of one stakeholder group, the same research may bear inspirational relevance in the eyes of another group of stakeholders.

\subsection{Switching between relevance types}

Aside from the fact that stakeholder groups can have different interpretations of the relevance of a given research, researchers also have the power to influence stakeholder groups' interpretations of relevance, or even to determine the relevance type(s) intended by their research. We believe there are some tenable practices which can be followed by researchers in order to achieve that goal. Here, we present three exemplar scenarios:

- Researchers might be able to switch between relevance types by changing their mode of research, or by complementing their current research mode by adopting additional compatible research modes. Such a switch between relevance types for instance can be observed in the case of two connected studies conducted by Dennis et al. [6] and Lee and Dennis [17]. In the former study, the authors are baffled with a particular result showing that, in their GSS controlled laboratory experiment, "GSS use inhibited information processing in more than half of the groups and inhibited information exchange in the rest” [6, p. 81]. In the latter study, Lee and Dennis [17] take a complementary hermeneutic approach and offer a plausible account as to why the laboratory experiment by Dennis et al. [6] went awry. While the study by Dennis et al. [6] could be argued to manifest cognitional relevance, one might consider that the study by Lee and Dennis [17] is conducive to acknowledging competing perspectives, and hence bears edificational relevance. The fact that relevance types can change as a result of change in research modes, among other things, implies that some research modes, compared to others, might be more suitable to achieve a certain type of relevance in a particular context. For instance, effectuational 
relevance might seem more likely to emerge in design research, rather than in survey-based research.

- $\quad$ Researchers might not manipulate their research mode or approach, but might still be able to have influence over the relevance type(s) that their research could bear. We suppose one scenario corresponding to such situation is that a researcher would take a follow-up inquiry step to collect indications of how his/her research has been able to further improve some stakeholders' lives in retrospect. We think one tenable way to take such a follow-up inquiry step is to conduct what is commonly known in research methodology texts as member checks.

- A third scenario of change in relevance type(s) could happen when a researcher neither manipulates his/her research mode(s) nor takes additional inquiry steps, but only provides additional informative details on his/her research design or results. For instance, in statistically conducted research, there might be situations where offering a discussion concerning the practical significance of some reported effect sizes, in addition to the conventional discussion of statistical significance, would shift a study from bearing cognitional relevance to bearing inspirational relevance in the eyes of certain stakeholder groups.

\subsection{Expansion of the typology of relevance}

There are several reasons behind our earlier argument that we do not make a parallel between the two concepts of authenticity and relevance. One major reason is that we consider the possibility that the number of relevance types may not remain limited to only four. In other words, we suppose that the typology of relevance offered above is open to further expansions. Such possibility mainly comes from the fact that the notion of empowerment entails the sufficient conceptual affordance to be further speculated upon. The speculation might lead to the identification of some more instantiations along the two dimensions of empowerment subject matter and empowerment focus. There is also the possibility that some more dimensions in addition to those two could be identified.

\section{Conclusion}

To produce only applicable or transferable knowledge is too limited a goal for IS research. We believe that the IS field should set its standards at a much higher level. Throughout this essay, we have argued that IS research must bear value in the eyes of its diverse stakeholders, but unlike the value perspective, our proposed perspective suggests that the source of value lies more in whether research stakeholders feel empowered by IS research rather than whether IS research questions or products hold distinctive scientific merits. In other words, we contend that to suitably empower its diverse stakeholder groups (including researchers themselves) is an indication of distinct scientific merits of IS research.

The empowerment perspective we put forward in this essay enables one to adopt a pluralist perspective of relevance. A particular significance of such pluralist view would be the promising potential it offers to build a more research-wise democratized IS community, where different research modes, practices and philosophies would gain the credit they deserve in terms of producing different types of relevant research. We maintain that the relevance types identified in this essay are equally important and each of them merit pursuit in IS research. Nevertheless, as the empowerment perspective implies, the identified relevance types must not necessarily be only invoked through academic papers and journals. Classrooms, newspaper articles, textbooks, corporate training sessions, and so forth are other venues offering an opportunity for IS research to empower its stakeholders.

There are several future research opportunities available of which we mention a few here. One productive avenue for future research would be to further investigate possible associations among different research modes and different types of relevance. In other words, future research can concentrate on the question of "what type(s) of relevance are more likely to emerge if one follows certain research modes or approaches?" Another future research area as implied earlier involves seeking to expand the typology of relevance to include as yet unidentified forms of empowerment effect. However, in a bigger picture, further research can also be pursued with the idea of "research on research relevance” in mind. This for instance could take the form of a comprehensive review of practicebased IS research published in journals and conferences, and comparing these to other types of IS research published in our top journals. Consistent with the philosophy underlying the notion of empowerment, future research might also attempt to devise solid and well-thought-out policies and practices designed to engage, and give power and decision-making roles to, different stakeholder groups in the process of conducting IS research. 
Although we earlier proposed that the relevancerigor dichotomy need not be so, and that relevance can coexist with rigor, we do suggest that the notion of what constitutes relevance needs to remain separate from our notions of methodological rigor and theoretical contribution. Pre-theoretical, atheoretical, and theory light research might all achieve high relevance. So too might research that does not employ the newest and most advanced methodological tools. However, so too can pure theory papers achieve high relevance as can research that focuses exclusively on the proper use of a particular method.

While the three concepts of methodological rigor, theoretical contribution and relevance are distinct from each other, the latter has received the least attention. We hope this essay encourages scholars to consider their purported relevance goals from their research and journal editors to consider means of evaluating research relevance that extend beyond downloads and citations to such considerations of how to promote and measure relevance while maintaining existing standards for method and theory.

\section{References}

[1] W.G. Astley and R.F. Zammuto, "Organization Science, Managers, and Language Games,” Organization Science (3:4), 1992, pp. 443-460.

[2] J.S. Benamati, M.A. Serva, D.F. Galletta, A. Harris, and F. Niederman, "The Slippery Slope of MIS Academia: A Discussion of the Quest for Relevance in Our Discipline," Communications of the Association for Information Systems 18, 2006, pp. 657-675.

[3] I. Benbasat and R.W. Zmud, "Empirical Research in Information Systems: The Practice of Relevance," MIS Quarterly (23:1), 1999, pp. 3-16.

[4] T.H. Davenport and M.L. Markus, "Rigor vs. Relevance Revisited: Response to Benbasat and Zmud," MIS Quarterly (23:1), 1999, pp. 19-23.

[5] A.R. Dennis, "Relevance in Information Systems Research," Communications of the Association for Information Systems 6, 2001, pp. 40-42.

[6] A.R. Dennis, K.M. Hilmer, and N.J. Taylor, "Information exchange and use in GSS and verbal group decision making: effects of minority influence,” Journal of Management Information Systems (14:3), 1997, pp. 61-88.

[7] K.C. Desouza, O.A. El Sawy, R.D. Galliers, C. Loebbecke, and R.T. Watson, "Beyond rigor and relevance towards responsibility and reverberation: Information systems research that really matters," Communications of the Association for Information Systems (17:1), 2006, pp. 341-353.

[8] M. Gallivan and A. Aryal, "Under What Conditions is IS Research Relevant to Practice? An Analysis of IS Scholars Who Are Mentioned or Cited Often in Trade Magazines and the General Media," Proceedings of the Eighteenth Americas Conference on Information Systems, 2012, Seattle, Washington, August 9-12.

[9] K.J. Gergen and T.J. Thatchenkery, "Organizational Science as Social Construction: Postmodern Potentials," Journal of Applied Behavioral Science (32), 1996, pp. 356377.

[10] G. Gill and A. Bhattacherjee, "Whom are we informing? Issues and recommendations for MIS research from an informing sciences perspective,” MIS Quarterly (33:2), 2009, pp. 217-235.

[11] V. Grover, "Value of IS Research: Let's Not Talk Crisis - but We Can Do Better," Communications of the Association for Information Systems (34:45), 2014, pp. 837-842.

[12] N.R. Hassan, "Value of IS Research: Is there a Crisis?" Communications of the Association for Information Systems (34:41), 2014, pp. 801-816.

[13] N.R. Hassan, J.F. Nunamaker Jr, I. Benbasat, R.O. Briggs, and B. Mueller, "Benefitting from IS Research-Who and How? A Panel on the Value of IS Research," Proceedings of the Nineteenth Americas Conference on Information Systems, 2013, Chicago, Illinois, August 1517.

[14] N. Kock, P. Gray, R. Hoving, H. Klein, M. Myers, and J. Rockart, "IS research relevance revisited: Subtle accomplishment, unfulfilled promise, or serial hypocrisy?," Communications of the Association for Information Systems (8:1), 2002, pp. 330-346.

[15] S. Kvale, "The social construction of validity," Qualitative Inquiry (1:1), 1995, pp. 19-40.

[16] A.S. Lee, "Rigor and Relevance in MIS Research: Beyond the Approach of Positivism Alone,” MIS Quarterly (23:1), 1999, pp. 29-33.

[17] A.S. Lee and A.R. Dennis, “A hermeneutic interpretation of a controlled laboratory experiment: a case study of decision-making with a group support system," Information Systems Journal (22:1), 2012, pp. 3-27.

[18] C.S. Levy, "Client self-determination,” in Handbook of clinical social work, 1983, pp. 904-919.

[19] Y.S. Lincoln and E.G. Guba, "But Is It Rigorous? Trustworthiness and Authenticity in Naturalistic Evaluation," In D.D. Williams (ed.), Naturalistic 
Evaluation, New Directions for Evaluation (30), 1986, San Francisco: Jossey-Bass.

[20] K. Manning, “Authenticity in Constructivist Inquiry: Methodological Considerations without Prescription," Qualitative Inquiry (3:1), 1997, pp. 93-115.

[21] S.A. Mohrman, C.B. Gibson, and A.M. Mohrman, "Doing Research That Is Useful to Practice: A Model and Empirical Exploration,” Academy of Management Journal (44:2), 2001, pp. 357-375.

[22] R.T. Mowday, "Presidential Address: Reaffirming Our Scholarly Values,” Academy of Management Review (22), 1997, pp. 335-345.

[23] A. Opie, "Qualitative research, appropriation of the 'other' and empowerment,” Feminist Review (40), 1992, pp. 52-69.

[24] D. Robey and M.L. Markus, "Beyond rigor and relevance: Producing consumable research about information systems,” Information Resources Management Journal (11:1), 1998, pp. 7-16.

[25] Rodwell, M.K., Social Work Constructivist Research, Taylor \& Francis, 1998.

[26] M. Rosemann and I. Vessey, "Toward improving the relevance of information systems research to practice: The role of applicability checks,” MIS Quarterly (32:1), 2008, pp. 1-22.

[27] Simon, B.L., The Empowerment Tradition in American Social Work: A History, Columbia University Press, 1994.

[28] D.W. Straub and S. Ang, "Editor's comments: Readability and the relevance versus rigor debate," MIS Quarterly (32:4), 2008, pp. iii-xiii.

[29] D.W. Straub and S. Ang, "Rigor and Relevance in IS Research: Redefining the Debate and a Call for Future Research,” MIS Quarterly (35:1), 2011, pp. iii-xi 